Excessive Internet Use: Psychological Vulnerability and Digital Literacy 1 
Excessive Internet Use: Psychological Vulnerability and Digital Literacy 2

\section{Excessive Internet Use by Young Europeans: Psychological Vulnerability and Digital Literacy?}

Ellen Johanna Helsper ${ }^{\text {** }}$ and David Smahel ${ }^{\mathrm{b}}$

${ }^{a}$ London School of Economics and Political Science, London, UK; ${ }^{b}$ Masaryk University, Brno, Czech Republic

Word count:7990

Corresponding author: Ellen Johanna Helsper, Media and Communications Department, London School of Economics and Political Science, WC2A 2AE London, UK, Tel: +44(0)2079556420, Email: e.j.helsper@1se.ac.uk , ORCID: 0000-0002-0852-2853

David Smahel, Faculty of Social Studies, Masaryk University, Joštova 10, Brno, 60200, the Czech Republic, Telephone number: +420 549497451 , Email:

smahel@fss.muni.cz, ORCID: 0000-0003-2767-4331

\section{Short biographies:}

Ellen Helsper is Associate Professor in the Media and Communications Department at the London School of Economics and Political Science. Her research interests include the links between social and digital inequalities; mediated interpersonal communication; and methodological innovation in media and communications research and is lead investigator on the From Digital Skills to Tangible Outcomes projects. She consults for governments, the third and commercial sector on participation in increasingly digital societies.

David Smahel, is Professor at the Institute of Children, Youth and Family Research, Masaryk University, the Czech Republic. He is a member of Interdisciplinary Research Team on Internet and Society which researches social-psychological implications of the Internet and technology. His current research focuses on adolescents' and adults' Internet use, the online risks of children and adolescents, the construction of online identities and virtual relationships, and Internet addiction. 
Excessive Internet Use: Psychological Vulnerability and Digital Literacy 3

Disclosure statement. No financial interest or benefit has arisen from the direct applications of this research.

Word count: 7551

Tables: 3

Figures: 2 


\title{
Excessive Internet Use by Young Europeans: Psychological Vulnerability and Digital Literacy?
}

\begin{abstract}
This paper combines clinical-psychological and digital literacy frameworks to shed new light on explanations for excessive Internet use (EIU). The combination of these opposing approaches leads to a more comprehensive explanation of intense use with negative outcomes. A survey with a random sample of 18,709 Internet-using children between 11 and 16 years old was carried out in 25 European countries. The study shows that there are interactional and indirect relationships between psychological and digital literacy variables and EIU. Psychologically vulnerable children with higher levels of digital engagement have the most negative outcomes while the least at risk are non-vulnerable children with high levels of literacy (interactional relationship). In reality, psychologically vulnerable children's risk of negative outcomes is exacerbated by their tendency to spend more time online but countered by their lower literacy levels (contradicting direct and indirect relationships). Among those who are not vulnerable, digital literacy is weakly related to negative outcomes. The implications of these results for future research are that explanations for EIU should incorporate psychological and digital literacy indicators. Practical implications are that clinical psychologists working with EIU should consider digital literacy in developing interventions and that digital inclusion interventions should consider the potential negative impact of increased Internet use on vulnerable young people. This paper's original contribution lies in showing that whether intense Internet use is related to negative outcomes depends on the psychological characteristics of the child.
\end{abstract}

\section{Excessive Internet Use by Young Europeans: Psychological Vulnerability and Digital Literacy?}

\section{Introduction}

The use of information and communication technologies (ICTs) increased exponentially amongst young people over the last decades, in terms of the number of devices used to 
access services and in terms of the actual time that children spend online. There is a duality in how this development is perceived. When discussed within a psychological and psychiatric framework, intense engagement is often associated with addiction and thus psychological problems. Most concern is directed at those children and young people who are seen to be at risk of excessive use (i.e. intense use that leads to negative outcomes) (Authors, 2009). However, within a digital literacy framework, broad ICT access, skills and high engagement levels are seen as an indicator of literacy, and thus as mostly positive. Research in this area shows that digital literacy is unequally distributed; socially vulnerable children are disadvantaged as regards their ability to take up the opportunities and avoid the risks available online (Paus-Hasebrink, Sinner \& Prochazka, 2014).

The two approaches come with different languages to describe the phenomenon of intense Internet use. The clinical-psychological approach describes the drivers underlying excessive use as needs based and link it to psychological vulnerability (Cao $\& \mathrm{Su}, 2007)$, while digital literacy frameworks have associated vulnerabilities based on socio-economic and demographic backgrounds with lower levels of digital skills and, subsequently, lower engagement with the Internet, preventing these young people from taking up the benefits of Internet use (Authors, 2013; Paus-Hasebrink et al., 2014; Sonck, Livingstone, Kuiper \& de Haan, 2011).

Thus, digital literacy theories generally consider intense use favorably because it indicates an increased uptake of services and learning opportunities (Authors, 2017), and the clinical-psychological framework is more negatively framed since it discusses intense use as an indicator of emotional or personal problems in a child's life (Cheng \& Peng, 2008). The aim of this paper is, therefore, to combine the clinical-psychological and digital literacy frameworks to shed new light on the complexity of the explanations for the negative outcomes of intense Internet use by children. In this discussion, it is 
important to distinguish intense and excessive Internet use because intense use is not always excessive, but it can instead be an indicator of high levels of literacy that allow the child to take up the myriad of opportunities available online. This paper uses the term excessive use to refer to intense use of the Internet that leads to negative outcomes.

This paper asks which combinations of socio-demographic, psychological, and digital literacy factors are most associated with excessive Internet use (EIU) amongst children. It hypothesizes that psychological deficits and digital literacy may have interactional, direct and indirect relationships with EIU that need to be included in analyses that aim to understand why some children experience negative outcomes from their intense engagement with the Internet and others are less likely to do so.

\section{A Psychological Framework for Excessive Use}

Under the clinical-psychological framework, the term excessive Internet use is often associated with pathologically intense Internet use and is, therefore, referred to as online addiction (Widyanto \& Griffiths, 2007), pathological Internet use (Morahan-Martin \& Schumacker, 2000), or Internet addiction disorder (Chou, Condron \& Belland, 2005). Such intense engagement with the Internet is usually defined through indicators originally designed to measure other types of addictive behavior (Widyanto \& Griffiths, 2007). Most studies of excessive use take a traditional psychological approach (i.e. Juvonen \& Gross, 2008) and relate it to social, mental, and physical impairments (Author, 2016).

The six components of excessive Internet use were developed and labelled Internet addiction to determine pathological intensive Internet use by Widyanto and Griffiths (2007). Based on Brown's (1993) concept of behavioral addictions, they argued that an Internet user is addicted if he or she experiences all the following aspects: salience, when the activity becomes the most important thing in an individual's life; mood change, or euphoria, when subjective experiences of the individual are 
significantly affected by the activity; tolerance, the process of requiring continually higher doses of the activity to achieve the original sensations; withdrawal symptoms, negative feelings which occur when unable to perform the activity or after termination of the activity; conflict, usually with the individual's closest social surroundings (family), but also accompanied with a significant decrease in school (or work) results or dropping out; relapse and reinstatement, the tendency to return to the damaging activity even after periods of relative control. Several different scales and factors have been proposed to measure EIU by other authors (e.g. Ko,. Yen, Chen, Chen \& Yen, 2005) but their basic premise is similar. While societal concern about addiction often lies with children, clinical-psychological research into this area often discusses these phenomena in older adolescents or adults (Festl, Scharkow \& Quand, 2013), college student samples in particular (Authors, 2015). This paper focusses on children from 11 to 16. This is important because while policy makers' and parental concern focuses on this age group there is little evidence on factors explaining negative outcomes of intense use that might inform policies or parental mediation for these young people (O'Neill et al., 2013).

Researchers critiqued the clinical psychological approach to describing outcomes of intense Internet use. Charlton and Danforth (2004) critique the broad brush approach often used in this area and argue that high engagement and addiction should be distinguished. The current practice in the field does not really allow for this, especially because, for many young people, high engagement with ICTs has become embedded in everyday life (Kardefelt-Winther, 2014b).

Under this clinical-psychological paradigm the outcome of intense use is seen as negative and is explained mostly through psychological vulnerabilities or deficits and the psychological characteristics of individuals (Cheng \& Peng, 2008). Cao and Su's (2007) research related Internet addiction to higher scores in neuroticism, psychosis, 
lying, emotional symptoms, and conduct problems. Ko, Yen, Yen, Lin and Yang (2007) have associated it with novelty or sensation seeking and with other offline behaviors, such as substance abuse, that are considered problematic (see also Yoo et al., 2004). On the other hand, Davis (2001) shows that individuals with lower self-efficacy can use the Internet to regain a positive evaluation of their own abilities. In research that focuses on young people, these psychological characteristics have in turn been related to sociodemographic characteristics.

Based on the existing longitudinal research (Buglass, Binder, Betts \& Underwood, 2017; Ko et al., 2009), this paper assumes that psychological characteristics are formed before young people start using the Internet and that, therefore, the causal relationship should be thought of with psychological characteristics leading to certain ways of engaging with the Internet leading to negative outcomes (i.e. excessive use). However, the causal direction of the relationships between psychological vulnerability, digital literacy and the negative and positive outcomes of Internet use can only truly be tested through longitudinal panel research or randomized controlled trials. Future research should test whether there is a feedback loop with psychological vulnerability leading to a reliance on the Internet to compensate for these deficits, thus leading to intense use with negative outcomes which might subsequently lead to more psychological problems. Or whether intense use in itself leads to negative outcomes and psychological vulnerability amongst children who did not have these before.

\section{Digital Literacy Frameworks for Intense Use}

In contrast with psychological studies, in studies of digital literacy, higher intensities of use are seen as a positive indicator because they indicate digital embeddedness and confidence in engaging with the opportunities available online (Authors, 2017). Digital literacy has been defined as relating to skills and different levels of engagement with the 
Internet and other ICTs (Authors, 2014; Van Dijk, 2005; Sonck et al., 2011). This framework explains intense use through the level of digital skills, the breadth of engagement (i.e. time spent online and number of activities undertaken), and the ubiquity of opportunities to connect (Van Deursen, 2010).

Here discussions about vulnerability in relation to intense Internet use are framed differently; individuals who are disadvantaged in a socio-economic or sociocultural sense are generally less skilled and have fewer opportunities to connect to the Internet in a meaningful way, which in turn leads to lower levels of engagement (Authors, 2012). Thus, vulnerabilities relate to less intense Internet use under this framework while personal vulnerabilities relate to a higher likelihood of intense use under the psychological framework.

The digital literacy framework explains different levels of engagement by linking things like educational background, gender, and ethnicity to differences in literacy levels. The link between socio-economic disadvantage and low engagement with ICTs is well established in the digital inclusion literature (Authors, 2012). The strongest predictors of high digital literacy are occupation, education, gender, and age (Authors, 2015; Witte \& Mannon, 2010). The digital literacy framework assumes that socio-demographic and socio-cultural factors are directly related to higher skills and more frequent engagement and that they are, through these mediators, indirectly related to intense engagement with a range of activities that can be found on the Internet (Authors, 2012). However, research shows that while skilled teenagers take up more opportunities than lower skilled teenagers, they are also more likely to be exposed to risks online (Authors, 2007). Recent research argues that more skilled youngsters with supportive parents take these risks but avoid the harm that might come from them (Paus-Hasebrink et al., 2014). 


\section{Bringing the Two Frameworks Together}

The previous discussion suggests a link between the clinical-psychological and digital literacy frameworks. While they offer different emphases in their explanations, both approaches focus on intense Internet use. Further similarities are that sociodemographic factors are part of both the clinical-psychological and the digital literacy approaches to intense use; under the first they are associated with particular psychological characteristics of the individual, and under the second they are related to the digital literacy levels of the individual. The clinical-psychological framework largely ignores how psychological characteristics interact with digital literacy and instead makes a direct link between psychological characteristics and intense and excessive use. While there is literature that looks at the link between literacy and risks (Blinka et al., 2015) and literacy and addiction (Leung \& Lee, 2012), this does not incorporate the psychological variables. The digital literacy framework, on the other hand, rarely incorporates clinical-psychological characteristics as an explanation of intense use and mostly ignores the fact that intense use could have negative implications for some children.

In Figure 1 models an integration of the two frameworks: socio-demographic characteristics are included as part of both the clinical-psychological and digital literacy approaches to intense Internet use. The factors commonly associated with intense use across these studies (i.e. gender, age, and education) are used to operationalize sociodemographic characteristics. In the model, self-efficacy, emotional problems, and sensation seeking were included because they are most consistently associated with excessive use in the clinical-psychological approach. The operationalization of the link between socio-demographic and digital literacy characteristics is derived from research on digital inclusion, where the most common definitions of high engagement encompass skills and breadth of use. As indicated earlier, age, gender, and education are 
most commonly (and directly) associated with higher levels of digital literacy under this framework.

[Figure 1]

Digital literacy research has argued that children with higher literacy levels should be able to take up the opportunities but avoid the negative outcomes that might result from the risks encountered during intense Internet use (Sonck et al., 2011), while the clinical psychological literature has argued that children with emotional problems are more at risk of negative outcomes of Internet use (i.e. excessive use).

Combining these two assumptions, Hypothesis 1 is that digital literacy and psychological problems interact so that children with high literacy levels and less psychological problems are least at risk of negative outcomes from intense Internet use, that is, excessive Internet use.

In addition, the literature suggests that disadvantaged children are more likely to have psychological problems but also that they have lower digital literacy levels in contrast to the addiction literature which argues that (psychologically) vulnerable children engage more intensely. It is unclear whether children who are psychologically vulnerable have higher or lower digital literacy levels under the broader definition of digital literacy applied in the literature on digital inclusion. We expect that in practice digital literacy has complex interaction effects in relation to offline vulnerability. Hypothesis 2 is, therefore, tentatively that in reality children with psychological problems are more digitally engaged (to escape their real life problems) and that this exacerbates the effect of psychological vulnerability on negative outcomes of EIU. 


\section{Materials and Methods}

\section{Sample}

The data of the EU Kids Online II study were used to test the hypotheses. This survey randomly sampled 1,000 Internet-using children in 25 European countries. This study was conducted in April/October 2010 across these countries and included 25,142 children (50\% girls) between 9 and 16 years old. Data were collected through face-toface, in-home surveys of participants, after initial pilot tests to ensure understanding. In each household, a child and one of his/her parents were asked about the child's online experiences. Following ESOMAR guidelines, informed consent from both parents and children was required for participation, and participants were assured of both confidentiality as well as anonymity. Only the data from the 11 - to 16 -year olds $(\mathrm{N}=$ 18,709) are analyzed in this paper, since younger children completed a shorter questionnaire that did not include questions about excessive Internet use.

\section{Measures}

\section{Socio-demographic Characteristics}

Gender (50\% boys $/ 50 \%$ girls $)$ and Age $(\mathrm{M}=13.54, \mathrm{SD}=1.68)$ : indicated by the child's parent.

Education. The ISCED classification of four educational categories (derived from parents' level of education) was used to create a 3 point scale: 1 'primary or less' (15\%), 2 'lower secondary or upper and post-secondary' (62.5\%), and 3 'tertiary education' $(22.5 \%)$.

\section{Psychological Characteristics}

All of the psychological characteristic items were answered using the following answer scale: 1 'not true', 2 'a bit true', and 3 'very true'. Scale scores were computed by 
averaging the items. To make sure that the operationalization of the different concepts and the answer scales were valid and reliable, and subject to the least possible socialdesirability bias, all of the items in the questionnaire were pilot tested through cognitive interviews with a representative subset of European children (see EUKidsOnline.net). Researchers from the EU and Global Kids Online networks have used these variables as scale variables and they have subsequently been used and tested in peer reviewed published research in this form by others (e.g. Blinka et al., 2015; Kardefelt-Winther, 2014b).

Sensation seeking $(\mathrm{M}=0.68 ; \mathrm{SD}=1.03 ; \alpha=.79)$. To assess the extent of sensation seeking, the items suggested by Stephenson et al. (2003) were adopted. Participants were asked: "How true is this of you?" with two items: 'I do dangerous things for fun' and 'I do exciting things, even if they are dangerous'.

Emotional problems $(\mathrm{M}=1.44 ; \mathrm{SD}=.38 ; \alpha=.69)$. Six items were used to capture participants' emotional problems. The items were adapted from the Strength and Difficulties Questionnaire (SDQ) (Goodman, 1999). Respondents were asked "How true is this of you?" with response alternatives (a) 'having a lot of headaches, stomachaches or sickness'; (b) 'being very angry and often losing their temper'; (c) 'often feeling unhappy, sad or tearful'; (d) 'being nervous in new situations and easily losing confidence'; (e) 'being easily distracted and finding it difficult to concentrate' and (f) 'having many fears, being easily scared'.

Self-efficacy $(\mathrm{M}=1.79 ; \mathrm{SD}=.47 ; \alpha=.65)$. The self-efficacy scale was adapted by taking the following four highest loading items from the Schwarzer and Jerusalem (1995) scale: a) 'It's easy for me to stick to my aims and achieve my goals'; b) 'I am confident that I can deal with unexpected problems'; c) 'f I am in trouble I can usually think of something to do'; and d) 'I can generally work out how to handle new situations'. 


\section{Digital Literacy and Engagement}

Skills. Internet skills were measured by summing the things that the child indicated being able to do on the Internet out of the following eight $(M=4.16, S D=2.67)$ : a) 'Compare different websites to decide if information is true'; b) 'Change filter preferences'; c) 'Bookmark a website (add to Favourites)'; d) 'Block unwanted adverts or junk mail/spam'; e) 'Delete the record of which sites which you visited'; f) 'Change privacy settings on a social networking profile'; g) 'Block messages from someone who you don't want to hear from'; and h) 'Find information on how to use the Internet safely'.

Internet self-confidence. Confidence was measured by asking children whether they thought that the statement 'I know lots of things about using the Internet' was 1 'not true', 2 'a bit true', or 3 'very true' $(M=2.27, S D=.66)$. This was the only item capturing the more qualitative element of skills related to confidence.

Time online. Time online operationalizes high levels of engagement, used in digital literacy studies, and measures intense use as is common in the Internet addiction literature. This purposefully separates intensity of use from negative outcomes of use. This was measured through two questions "About how long do you spend using the Internet in a normal non-school day/in a normal school day?" The answer categories for both questions were categorical, ranging from 1 'just a few minutes' to 10 'more than four hours'. The score for average school-day use was multiplied by 5 and added to the score for average non-school-day use, which was multiplied by 2 . The resulting scores (ranging between 0 and 76) were recategorized into a scale from 1 'none at all', 2 'less than 3.5 hrs a week' to 6 'more than 21 hrs a week' $(\mathrm{M}=3.87, \mathrm{SD}=1.15)$.

Breadth of engagement. This was measured by calculating the number of activities that young people undertook online out of 17 activities they had undertaken in the past 
month $(M=7.02, S D=3.02)$ (Hasebrink, 2012). Sample activities included 'watched video clips', 'downloaded a movie', or 'used a webcam.'

\section{Excessive Internet Use (EIU)}

The five questions used in the present study were derived from the 10 -item version of Widyanto and Griffiths' (2007) 6 factor scale measuring symptoms of problematic Internet use (Authors, 2009; Authors, 2011). One question capturing mood changes addressed two factors: euphoria and withdrawal symptoms. Participants answered how often, in the previous 12 months, they had gone without eating or sleeping because of the Internet (salience); how often they felt bothered when they could not be online (euphoria and withdrawal symptoms); how often they caught themselves surfing when they had not really been interested (tolerance); whether they dedicated less time to their family, friends, or schoolwork because of the time spent on the Internet (conflict); and whether they tried to spend less time on the Internet without success (relapse and reinstatement). Four-point response scales were offered: 1 'never/almost never', 2 'not very often', 3 'fairly often', and 4 'very often'. Scale scores consisted of the average score out of five items; a higher score indicated more problematic Internet use $(M=1.66$; $\mathrm{SD}=1.61 ; \alpha=.78)$.

\section{Analytical Approach}

A two-fold analysis of Internet use tested the interaction effects and indirect relationships between psychological characteristics and digital literacy and EIU. First, a linear regression of EIU was conducted whereby the relevance of socio-demographic explanations (part of both the psychological and digital inclusion frameworks), clinicalpsychological approaches, and digital literacy frameworks were tested. The linear regressions allow for testing of interactions between the psychological and digital literacy indicators in relation to EIU (H1). This was done to understand whether, for 
example, digital literacy has different implications for those who are psychologically vulnerable than for those who are psychologically stronger. The linear regressions tested for interaction relationships with skills only because these are considered the key element within digital literacy theory and are what most digital inclusion interventions focus on. Skills allow individuals to avoid negative outcomes while simultaneously being able to take up the opportunities afforded by intense use.

The second part of the analysis consisted of a path analysis which examined the direct and indirect relationships between psychological and digital inclusion indicators and EIU ${ }^{\mathrm{i}}$. This looked into whether in reality digital literacy is unequally distributed amongst the psychologically vulnerable and non-vulnerable (H2). This analysis allows for an understanding of where interventions might be best placed, since the path analysis tests how digital literacy is distributed in reality and whether it exacerbates or weakens negative outcomes of Internet use, presuming the causal sequence based on the literature and related empirical research (see Figure 1).

\section{Results}

\section{Independent and Interaction Effects}

The linear regression of EIU showed significant contributions of socio-demographic, psychological characteristics and digital literacy characteristics. The interaction terms between psychological characteristics and digital literacy were also significant (see Table 1).

[Table 1]

Amongst the socio-demographic variables, education was the only significant predictor; children from lower educated households were more at risk of excessive Internet use. All psychological characteristics and digital literacy characteristics, with the exception of the number of platforms the child uses, were significantly related to 
EIU. Standardized effect sizes were largest for digital skills (negatively related to EIU), time spent online, the interactions between emotional problems and Internet skills, and between self-efficacy and Internet skills.

Figure 2 shows the predicted values of EIU for children with different skill levels by the level of emotional problems and self-efficacy; the two interactions were significant in the linear regression. Since the interaction between sensation-seeking and skills was not significant, this is not depicted graphically.

Those most at risk of negative outcomes are those with high levels of emotional problems or low self-esteem and high skill sets. Those least at risk of negative outcomes are those with low levels of emotional problems and a high skill set, and those with a high skill set and high self-efficacy.

[Figure 2]

\section{Direct and Indirect Effects}

The linear regressions do not indicate whether digital literacy is unequally distributed between young people with psychological vulnerabilities and those without. They only indicate the direct relationship between skills and psychological vulnerabilities and outcomes and the level of negative outcomes children with different combinations of skills and vulnerabilities experience. That is, interactions in linear regressions show what the level of negative outcomes of intense use is for children with different levels of skills but not whether children with certain characteristics are actually more likely to have certain skill levels and whether this currently leads to higher or lower excessive Internet use amongst those with psychological vulnerabilities. Therefore, a path analysis was conducted using the model as presented in Figure 1. Since the EU Kids Online II data were derived from a cohort study, this order was based on the theoretical assumptions reflected in Figure 1 and on the longitudinal research which shows that psychological characteristics come before Internet use (e.g. Buglass, 
Binder, Betts and Underwood, 2017; Ko et al., 2009) and, similarly, from longitudinal research that shows that skills and literacy are stronger predictions of engagement than engagement is of skills and literacy (Authors, 2016).

The model as tested in Table 2 has a good fit (see $\mathrm{Hu} \&$ Bentler, 1995, 1999) under all indicators for complex models $\left(\chi^{2}(5)=46.94, \mathrm{p} .=.00 ; \mathrm{CFI}=.999 ; \mathrm{RMSEA}=.02\right.$ (c.i.=.01-.02), $\mathrm{p}=1 ; \mathrm{AIC}=166.94 ;) ; 23 \%$ of the variance in EIU is explained by the model as presented in Table 2 .

A model that excludes the psychological variables is a slightly worse fit $\left(\chi_{(5)}^{2}=71.796, p .=.00 ; \mathrm{CFI}=.997 ; \mathrm{RMSEA}=.02\right.$ (c.i.=.02-.03), $\mathrm{p}=1 ; \mathrm{AIC}=131.796$; $\left.\mathrm{R}^{2}=.17\right)$ as is a model that excludes the Internet use variables $\left(\chi^{2}(4)=69.195, \mathrm{p} .=.00\right.$; $\mathrm{CFI}=.992 ; \mathrm{RMSEA}=.03$ (c.i. $\left.=.02-.03), \mathrm{p}=1 ; \mathrm{AIC}=131.195 ; \mathrm{R}^{2}=.15\right)$.

In most cases, the path model shows similar results to the linear regression but there were some interesting differences. While in the linear regression model household education levels were independently and significantly related to excessive use at the $\mathrm{p}<.05$ level, the effect of education on EIU was shown in the path model to be completely indirect and mostly mediated through its relationship with self-efficacy. Gender, not significant in the linear regression, was directly and significantly, if weakly, related to EIU. Age, also not significant in the linear regressions, was strongly but mostly indirectly related to EIU. Emotional problems and time spent online had the strongest total relationships with EIU after age. In what follows, the direct and indirect paths from the different socio-demographic and the psychological indicators to EIU are discussed, followed by the direct paths from digital inclusion variables to EIU (see Tables $2 \& 3)$. 


\section{[Tables $2 \& 3]$}

\section{Socio-Demographic Characteristics}

Gender was significantly related to all psychological variables; boys were higher on sensation seeking and self-efficacy and reported less emotional problems. Similarly, boys indicated spending more time online and higher Internet skill levels even if their use of the Internet was not broader. The direct relationship between gender and EIU is quite weak $(b=.06 ; \beta=.02)$ and since the indirect relationships are similarly small $(b=.08 ; \beta=.02)$ compared to that of other socio-demographic variables, the total effects of gender on EIU are also small $(b=.13 ; \beta=.04)$.

Age has direct positive relationships with the psychological variables; older children are better off psychologically than younger children. Strong positive relationships were also found with digital literacy; age is related to the Internet being more embedded in everyday life. The direct relationship between age and EIU was not as strong $(b=.05 ; \beta=.07)$. The strong mediating effects of other variables make age the variable with one of the strongest total effects on EIU $(b=.19 ; \beta=.26)$.

The educational level of the household of the child has positive but weak relationships with all psychological and digital literacy characteristics. Children in households with higher education are better off psychologically and the Internet is more embedded in their everyday lives. Education's strongest relationship is with selfefficacy. Education was not significantly directly related to EIU and thus the total effects were amongst the weakest $(b=.05 ; \beta=.02)$.

\section{Psychological Characteristics}

Children with higher self-efficacy levels had lower levels of EIU $(b=-.13 ; \beta=-.04)$, while children who had more emotional problems $(b=.84 ; \beta=.19)$ and were higher in sensation seeking $(b=.20 ; \beta=.13)$ had higher levels of EIU. In addition to these direct 
effects, the relationships between psychological characteristics and EIU were mediated by digital literacy characteristics. The indirect relationships between sensation seeking $(b=.07 ; \beta=.04)$, self-efficacy $(b=.13 ; \beta=.04)$, and emotional problems $(b=.04 ; \beta=.01)$ and EIU are all positive. Self-efficacy and sensation seeking were both positively related to time spent online, skills, and breadth of engagement, while emotional problems were positively related to time spent online and breadth of engagement but negatively to Internet skills. That is, children who are high in self-efficacy and sensation seeking use the Internet more and are more skilled, while children who have more emotional problems use the Internet more but have fewer skills.

The combination of direct and indirect relationships makes emotional problems one of the strongest predictors of EIU $(b=.88 ; \beta=.20)$. Most of this relationship is direct. Sensation seeking also has a relatively large total effect on EIU $(b=.27 ; \beta=.17)$ but a quarter of this effect is indirect. Self-efficacy has an insignificant total effect $(b=.002$; $\beta=.001$ ) because the indirect and direct effects cancel each other out.

\section{Digital Literacy Characteristics}

All digital literacy and engagement variables were positively related to EIU. That is, children who indicated having more Internet skills $(b=.06 ; \beta=.10)$, those who spend more time online $(b=.26 ; \beta=.19)$ and those used the Internet more broadly $(b=.06 ; \beta=$ .12 ), were more likely to experience negative outcomes of Internet use.

\section{Comparing Indirect and Direct Effects}

Age was the strongest predictor of EIU but this was mostly indirect, mediated by its strong relationship with sensation seeking, more time spent online, and broader engagement with the Internet. The direct and indirect relationships between gender, education and EIU were relatively weak after controlling for the relationships between psychological characteristics, digital literacy and EIU. For education levels, in 
particular, it is interesting to note how the positive relationships with the different psychological characteristics counter the positive relationships with literacy.

The results show that emotional problems are a direct concern for EIU. The relationship between higher emotional problems and EIU is mostly direct. Nevertheless, the indirect relationships are interesting because they cancel each other out; those with more emotional problems are less skilled and less at risk of EIU but also spend more time online and through this more at risk of EIU.

Sensation seeking is directly and indirectly related to higher EIU. A large part of this relationship is mediated by higher levels of literacy, exacerbating the higher levels of EIU of those who have higher levels of sensation seeking.

Low levels of psychological self-efficacy were directly related to higher levels of EIU. However, lower levels of self-efficacy were also related to lower levels of Internet embeddedness which subsequently was related to lower EIU. The magnitudes of these countervailing direct and indirect effects were similar, balancing each other out and leading to a neutral total relationship between self-efficacy and EIU.

\section{Discussion}

This paper shows a combination of digital literacy and psychological vulnerability frameworks is necessary to explain negative outcomes of intense use (i.e. excessive Internet use - EIU). Clinical-psychological studies often ignore how digital literacy interacts with and augments psychological deficits in relation to EIU. Equally problematic is that digital literacy studies mostly ignore psychological vulnerabilities of children in relation to potentially negative outcomes of intense engagement. The three strongest predictors of EIU fall in different categories: amongst the socio-demographic variables this is age; amongst the psychological variables this is emotional problems; and amongst the digital literacy variables this is, unsurprisingly, the time spent online though, surprisingly, skills also had this effect for vulnerable young people. It is thus 
not enough to see these frameworks merely as different explanations of excessive Internet use, it is in their combination and interaction that their strength comes to the fore.

\section{Combining Psychological and Digital Literacy Frameworks}

H1 was that digital literacy and psychological problems interact so that children with high literacy levels and less psychological problems are least at risk of EIU. This was largely supported. However, unexpectedly children with high literacy levels and psychological problems were more at risk of EIU. When controlling for everything else, including the interactions, those with higher skill levels had fewer negative outcomes. Thus, in accordance with the predictions from our research on digital literacy, digital skills on their own are related to the ability to avoid negative outcomes. However, when digital skills are placed in interaction with indicators of vulnerability, such as emotional problems, higher literacy levels were related to more negative outcomes.

$\mathrm{H} 2$ asked whether in reality offline vulnerability is directly as well as indirectly (through digital literacy) related to EIU. That is, whether children who have psychological problems have higher engagement levels which exacerbate the negative outcomes they experience. This was supported for all psychological variables considered (i.e. sensation seeking, self-efficacy, and emotional problems) but some of the effects were contradictory where lower skill levels amongst psychologically vulnerable young people were related to lower, and more time spent online was related to higher levels of EIU.

This paper revealed that age and education are strongly related to EIU but that this relationship is not direct as assumed in other studies conducted with adults (see also Authors, 2011). Understanding which factors associated with sensation seeking put older children more at risk of EIU could help these young people avoid negative outcomes while still enabling them to take up online opportunities. 
This paper further showed that children who are high sensation seekers might be better able to fulfill their needs online when they have sufficient skills and are, therefore, more at risk of EIU. Children with lower levels of digital literacy might be unable to fulfill their sensation seeking needs online and undertake risky activities offline instead. This suggests that compensation needs might have positive effects, as shown in Valkenburg and Peter's (2007) study, but also negative effects as brought up in this study.

This paper puts previously found associations between emotional problems and EIU (Ko et al, 2007; Cheng \& Peng, 2008) into context. Emotional problems are most important because they have both direct and indirect relationships with EIU. While in regression analyses skills interacted with emotional problems so that higher skills amongst children with emotional problems related to higher EIU, the path analysis showed that children with emotional problems are less digitally skilled. Controversially, this could mean that interventions that increase digital skills amongst young people with high levels of emotional problems could put these children at high risk of EIU, especially since they tend to spend more time online.

Caution is needed when drawing causal conclusions about indirect effects. Most of the EIU data available at the moment, including that used for this study, are cohort based and thus do not allow for causal conclusions. To study this in detail, more longitudinal research with children is needed to understand the temporality, causality and cyclical nature of processes in the relationship between digital literacy and the negative and positive outcomes of this use. The question remains whether intense Internet use leads to psychological problems or whether, as is assumed here, it is the psychological problems that come first, get exacerbated by increased Internet use and skills, and eventually lead to negative outcomes which might then lead to more 
psychological problems. The suggestion is that emotional problems should be recognized and be dealt with to prevent increased literacy from having this effect.

\section{Digital Literacy}

Those working under the digital literacy paradigm are advised to consider the effects of psychological characteristics. For those coming from a digital literacy perspective it would otherwise be hard to understand why digital literacy characteristics were positively related to EIU. That children who are more skilled are also more likely to encounter negative outcomes can be explained by referring back to the interactions with the psychological characteristics found in the linear regressions and the additive relationships from the path analyses presented in this paper.

The positive relationships of time online and breadth of engagement with EIU indicate that the digital literacy approaches should be considering negative outcomes of intense use as well as opportunities. Researchers should aim to clarify why higher literacy amongst vulnerable children is linked to negative outcomes and whether, through longitudinal and causal research with this particular group, there is a negative spiral between high intensity of use, negative outcomes, and well-being. Incorporating psychological vulnerabilities allows digital literacy researchers and practitioners to draw conclusions about the optimal levels of engagement with the technology and move away from a model that has looked at more intense engagement and higher skills as a mostly positive phenomenon (e.g. Van Deursen, 2010; Van Dijk, 2005). By contextualizing it in the offline realities of the individuals, they strengthen their argument and effectiveness of training by including offline vulnerabilities that go beyond variations between individuals with different socio-demographic backgrounds (see also Authors, 2012). Important is that this study confirms that children who have more positive engagement with the Internet are also more likely to have negative 
experiences (Authors, 2010), but that the latter is especially the case for emotionally vulnerable young people.

\section{Psychological Frameworks}

The results presented in this paper suggest that for researchers working within the clinical-psychological frameworks it is useful to take into account the digital literacy approach which allows for a more complete explanation of EIU. These researchers and practitioners focusing on addiction often do not include more complex Internet use characteristics. Internet use characteristics are controlled for but not seen in conjunction with psychological characteristics. The role that Internet skills play in interaction with the psychological variables points towards the need to adjust the clinical-psychological model even when the psychological characteristics of the child play a significant independent role as well. In a clinical setting, treating psychological deficits on their own will not be sufficient, nor will a focus on limiting engagement with the Internet. Understanding which types of digital literacy are likely to amplify specific types of psychological vulnerabilities when it comes to Internet use, can lead to a more nuanced theorization and avoid the idea that intense use in itself is always negative (KardefeltWinther, 2014b).

\section{Conclusions}

This paper contributes to the clinical-psychological approach to understanding intense Internet use by arguing that Internet use and skills characteristics of the child should be taken into consideration to understand why some children are more at risk of experiencing negative outcomes of Internet use. Including these factors increases the level to which excessive use, that is, intense Internet use with negative outcomes, can be explained significantly. On the other hand, the digital literacy approach, which considers intense Internet use as a mostly positive phenomenon, should take the 
findings from clinical-psychological research into consideration. A contribution of this paper in combining these frameworks, is to show that increasing digital literacy amongst psychologically vulnerable young people, who tend to have lower skill levels but higher intensities of use, might put them at a higher risk of negative outcomes of this intense use. Interventions and researchers need to keep this interaction in mind and be aware of child's context when designing digital literacy training around taking up the opportunities and avoiding the risks of being online.

A discussion is needed about what optimal levels of use and acceptable levels of risk are, instead of assuming that all negatively perceived consequences should be avoided. Some risks need to be run for children to develop coping strategies or to develop themselves as resilient, independent individuals during their teenage years. In this paper, the choice was made to see skills and the time spent online as explanations of excessive Internet use. But this relationship might be reversed - those who have run more risks and have had problems in the past have developed the skills to deal with them later on and engage more intensely with the Internet. Similarly, this paper did not look at motivations for spending more time online or for engaging with the Internet in broader ways. Kardefelt-Winther (2014a) has argued that it is only people who have certain types of (compensatory) motivations in combination with certain types of psychological vulnerabilities that intense use will turn into persistent excessive use (i.e. leading to negative offline outcomes).

\section{Future Research}

Skills questions have improved since the EU Kids Online survey was done and the project partners have proposed a revision which allows for testing of the relationships of different types of literacy with EIU, which was not possible with the current dataset (Authors, 2014). 
Future research should incorporate a more nuanced, theoretically grounded, broader set of questions around digital literacy to understand whether certain types of literacy and engagement are more likely to lead to negative outcomes for the psychologically vulnerable.

Future research should focus on separately studying children at risk of temporary excessive Internet use and those suffering from more extreme, enduring forms of addiction. The development of a verified clinical scale of excessive Internet use or online addiction is needed to be able to differentiate the levels of excessive Internet use and its consequences. This is especially important in light of this study's findings of a strong relationship between Internet skills and excessive use, which suggests that there is a confounding of concepts which might be partly explained by the non-inclusion of motivations for use in this model and the temporality of compensatory behaviors. This paper cannot confirm this, but it can be argued that the indicators on the excessive use scale measure temporary intense, embedded use and not pathological use or Internet addiction. Future research should incorporate different compensatory motives to understand which types of intense use for which types of young people converts temporary excessive use into a more problematic, long-term negative engagement with the Internet. 


\section{References}

Publications by Authors (2007, 2009, 2010, 2011, 2012, 2013, 2014, 2015, 2016, 2017) anonymized for review purposes

Blinka L., Škařupová K., Ševčíková A., Wölfling K., Müller, K.W., \& Dreier, M. (2015). Excessive Internet use in European adolescents: what determines differences in severity?. International Journal of Public Health, 60, 249-56. doi:10.1007/s00038-014$0635-\mathrm{x}$

Brown, R.I.F. (1993). Some contributions of the study of gambling to the study of other addictions. In W.R. Eadington, and J.A. Cornelius (Eds.), Gambling behavior and problem gambling, (pp. 241-72). Reno: Institute for the Study of Gambling and Commercial Gaming, University of Nevada.

Buglass, S.L., Binder, J.F., Betts, L.R., \& Underwood, J.D.M. (2017) Motivators of online vulnerability: The impact of social network site use and FOMO. Computers in Human Behavior, 66, 248-255.doi: 10.1016/j.chb.2016.09.055

Cao, F., \& Su, L. (2007). Internet addiction among Chinese adolescents: prevalence and psychological features. Child: Care, Health and Development, 33, 275-81. doi:10.1111/j.1365-2214.2006.00715.x

Charlton, J. P., \& Danforth, I.D.W. (2004). Differentiating computer-related addictions and high engagement. In J. Morgan, C.A. Brebbia, J. Sanchez, \& A. Voiskounsky (Eds.), Human perspectives in the Internet society: Culture, psychology, gender, (pp. 59-68). Southampton: WIT Press.

Cheng, Y. F., \& Peng, S. S. (2008). University Students' Internet Use and Its Relationships with Academic Performance, Interpersonal Relationships, Psychosocial Adjustment, and Self-Evaluation. Cyberpsychology and Behavior, 2, 467-9. doi:10.1089/cpb.2007.0128 
Chou, C., Condron, L., \& Belland, J. (2005). A Review of the Research on Internet Addiction. Educational Psychology Review, 17, 363-388. doi:10.1007/s10648-005$8138-1$

Davis, R. A. (2001). A cognitive-behavioral model of pathological Internet use. Computers in Human Behavior, 17, 187-95. doi:10.1016/S0747-5632(00)00041-8

Festl, R., Scharkow, M., \& Quand, T. (2013). Problematic computer game use among adolescents, younger and older adults. Addiction, 108, $592 \quad-\quad 599$. doi:10.1111/add.12016

Goodman, R. (1999). The extended version of the Strengths and Difficulties Questionnaire as a guide to child psychiatric caseness and consequent burden. Journal of Child Psychology and Psychiatry, 40, 791-799. doi:10.1017/S0021963099004096 Hasebrink, U. (2012). Young Europeans' online environments: a typology of user practices. In S. Livingstone \& A. Goerzig (Eds.), Children, risk and safety on the Internet, (pp. 127-139). Bristol: The Policy Press.

Hu, L., \& Bentler, P. M. (1995). Evaluating model fit. In R.H. Hoyle (Ed.), Structural equation modeling: Concepts, issues, and application, (pp. 76-99). Thousand Oaks: Sage Publications.

Hu, L., \& Bentler, P. M. (1999). Cut-off criteria for fit indexes in covariance structure analysis: Conventional criteria versus new alternatives. Structural Equation Modelling: A Multidisciplinary Journal, 6, 1-55. doi:10.1080/10705519909540118 Juvonen, J., \& Gross, E. (2008). Extending the school grounds? Bullying experiences in cyberspace. Journal of School Health, 78, 496-505. doi:10.1111/j.17461561.2008.00335.x

Kardefelt-Winther, D. (2014a). A conceptual and methodological critique of Internet addiction research: towards a model of compensatory Internet use. Computers in Human Behavior, 31, 351-354. doi:10.1016/j.chb.2013.10.059 
Kardefelt-Winther, D. (2014b). The moderating role of psychosocial well-being on the relationship between escapism and excessive online gaming. Computers in Human Behavior, 38, 68-74. doi:10.1016/j.chb.2014.05.020

Ko, C.-H., Yen, J.-Y., Chen, C.-C., Chen, S.-H., \& Yen, C.-F. (2005). Proposed diagnostic criteria of Internet addiction for adolescents. Journal of Nervous and Mental Disease, 193, 728-733. doi:10.1097/01.nmd.0000185891.13719.54

Ko, C.-H., Yen J.-Y., Yen C.-F., Lin, H.-C., \& Yang, M.-J. (2007). Factors predictive for incidence and remission of Internet addiction in young adolescents: A prospective study. Cyber Psychology \& Behavior, 10, 545-551. doi:10.1089/cpb.2007.9992

Ko, C.-H., Yen J.-Y., Chen, C.-S., Yeh, Y.-C. \& Yen, C.-F. (2009). Predictive values of psychiatric symptoms for Internet addiction in adolescents: a 2-year prospective study. Archives of Pediatrics \& Adolescent Medicine, 163, 937-943. doi:10.1001/archpediatrics.2009.159

Leung, L., \& Lee, P. S.N. (2012). Impact of Internet Literacy, Internet Addiction Symptoms, and Internet Activities on Academic Performance. Social Science Computer Review, 30, 403-418. doi:10.1177/0894439311435217

Morahan-Martin, J. M., \& Schumacker, P. (2000). Incidence and correlates of pathological Internet use. Computers in Human Behavior, 16, 13-29. doi:10.1016/S0747-5632(99)00049-7

O'Neill, B. Strakstrud, E., \& McLaughlin, S (2013). Towards a better Internet for Children: Policy Pillars, Players and Paradoxes. Goteborg: Nordicom.

Paus-Hasebrink, I., Sinner, P., \& Prochazka, F. (2014). Children's online experiences in socially disadvantaged families: European evidence and policy recommendations. London: The London School of Economics and Political Science, Department of Media and Communication. 
Schwarzer, R., \& Jerusalem, M. (1995). Generalized self-efficacy scale. In J. Weinmann, S. Wright \& M. Johnston (Eds.), Measures in health psychology: A user's portfolio. Causal and control beliefs (pp. 35-37). Windsor: NFER-Nelson.

Sonck, N., Livingstone, S., Kuiper, E., \& de Haan, J. (2011). Digital literacy and safety skills. London: LSE, EU Kids Online.

Stephenson, M. T., Hoyle, R. H., Palmgreen, P., \& Slater, M. D. (2003). Brief measures of sensation seeking for screening and large-scale surveys. Drug and Alcohol Dependence, 72(3), 279-286. doi:https://doi.org/10.1016/j.drugalcdep.2003.08.003

Valkenburg, P., \& Peter, J. (2007). Preadolescents' and adolescents' online communication and their closeness to friends. Developmental Psychology, 43, 267-277. doi:10.1037/0012-1649.43.2.267

Van Deursen, A.J.A.M. (2010). Internet skills, vital assets in an information society. Enschede: Twente University.

Van Dijk, J. A. G. M. (2005). The deepening divide: Inequality in the information society. Thousand Oaks: Sage.

Widyanto, L., \& Griffiths, M. (2007). Internet addiction: does it really exist? (revisited). In J. Gackenbach, (Ed.), Psychology and the Internet: Intrapersonal, interpersonal, and transpersonal implications (pp. 141-63). San Diego: Academic Press.

Witte, J.C. \& Mannon, S.E. (2010). The Internet and Social Inequalities. London: Routledge.

Yoo, H. J., Cho, S. C., Ha, J., Yune, S. K., Kim, S. J., Hwang, J., ... Lyoo, I. K. (2004). Attention deficit hyperactivity symptoms and Internet addiction. Psychiatry \& Clinical Neurosciences, 58, 487-494. doi:10.1111/j.1440-1819.2004.01290.x 
Table 1 Linear regression of excessive Internet use (indicator number of negative outcomes out of 5)

\begin{tabular}{|c|c|c|c|c|c|}
\hline & $\mathrm{b}$ & $\mathrm{SE}(\mathrm{b})$ & $\beta$ & $\mathrm{T}$ & Sig. \\
\hline Gender (Boy) & -.02 & .02 & -.01 & -.76 & .45 \\
\hline Age & .00 & .01 & .00 & -.12 & .91 \\
\hline Education (1-3) & $-.08 * *$ & .02 & -.08 & -4.37 & .00 \\
\hline Sensation seeking & $.20 * *$ & .02 & .11 & 8.16 & .00 \\
\hline Self-efficacy ${ }^{a}$ & $-.24 * *$ & .04 & -.19 & -6.12 & .00 \\
\hline Emotional problems & $.28 * *$ & .05 & .18 & 5.42 & .00 \\
\hline Internet skills & $-.20 * *$ & .02 & -.45 & -10.64 & .00 \\
\hline Internet confidence & $.05 * *$ & .02 & .05 & 2.48 & .01 \\
\hline Time online & $.25 * *$ & .01 & .45 & 24.11 & .00 \\
\hline Breadth of engagement & $.06 * *$ & .00 & .19 & 13.83 & .00 \\
\hline Self-efficacy ${ }^{*}$ Skill & $.06^{* *}$ & .01 & .24 & 7.23 & .00 \\
\hline Sensation seeking*Skill & .00 & .00 & .01 & .69 & .49 \\
\hline Emotional problems*Skill & $.10 * *$ & .01 & .34 & 9.61 & .00 \\
\hline
\end{tabular}

Base: All Children, aged 11-16

a Self-efficacy scale is reversed; a higher score indicates lower self-efficacy.

$* \mathrm{p}<.05 * * \mathrm{P}<.01$ 
Table 2 Coefficients path model negative outcomes of excessive Internet use (EIU)

\begin{tabular}{|c|c|c|c|c|c|}
\hline & & & $\mathrm{b}$ & $\mathrm{SE}(\mathrm{b})$ & $\beta$ \\
\hline Age & $-->$ & Sensation seeking & 0.08 & 0.00 & 0.17 \\
\hline Age & $-->$ & Self-efficacy & 0.05 & 0.00 & 0.23 \\
\hline Age & $-->$ & Emotional problems & -0.01 & 0.00 & -0.04 \\
\hline Age & $-->$ & Skills & 0.60 & 0.01 & 0.49 \\
\hline Age & $-->$ & Time online & 0.19 & 0.00 & 0.36 \\
\hline Age & $-->$ & Breadth of engagement & 0.70 & 0.01 & 0.45 \\
\hline Age & $-->$ & EIU & 0.05 & 0.01 & 0.07 \\
\hline Gender (boys) & $-->$ & Sensation seeking & 0.37 & 0.01 & 0.18 \\
\hline Gender (boys) & $-->$ & Self-efficacy & 0.05 & 0.01 & 0.05 \\
\hline Gender (boys) & $-->$ & Emotional problems & -0.06 & 0.01 & -0.08 \\
\hline Gender (boys) & $-->$ & Skills & 0.10 & 0.03 & 0.02 \\
\hline Gender (boys) & $-->$ & Time online & 0.09 & 0.01 & 0.04 \\
\hline Gender (boys) & $-->$ & Breadth of engagement & 0 & 0 & 0 \\
\hline Gender (boys) & $-->$ & EIU & 0.06 & 0.02 & 0.02 \\
\hline Education & $-->$ & Sensation seeking & 0.07 & 0.01 & 0.04 \\
\hline Education & $-->$ & Self-efficacy & 0.10 & 0.01 & 0.12 \\
\hline Education & $-->$ & Emotional problems & -0.04 & 0.00 & -0.07 \\
\hline Education & $-->$ & Time online & 0.11 & 0.01 & 0.05 \\
\hline Education & $-->$ & Skills & 0.37 & 0.03 & 0.08 \\
\hline Education & $-->$ & Breadth of engagement & 0.35 & 0.03 & 0.06 \\
\hline Education & $-->$ & EIU & 0 & 0 & 0 \\
\hline Self-efficacy & $-->$ & Time online & 0.10 & 0.02 & 0.04 \\
\hline Self-efficacy & $-->$ & Skills & 1.02 & 0.04 & 0.17 \\
\hline Self-efficacy & $-->$ & Breadth of engagement & 0.75 & 0.04 & 0.10 \\
\hline Self-efficacy & $-->$ & EIU & -0.13 & 0.02 & -0.04 \\
\hline Emotional problems & $-->$ & Time online & 0.13 & 0.02 & 0.04 \\
\hline Emotional problems & $-->$ & Skills & -0.19 & 0.05 & -0.03 \\
\hline Emotional problems & $-->$ & Breadth of engagement & 0.28 & 0.05 & 0.03 \\
\hline Emotional problems & $-->$ & EIU & 0.84 & 0.03 & 0.19 \\
\hline Sensation seeking & $-->$ & Time online & 0.09 & 0.01 & 0.08 \\
\hline Sensation seeking & $-->$ & Skills & 0.27 & 0.02 & 0.10 \\
\hline Sensation seeking & $-->$ & Breadth of engagement & 0.46 & 0.02 & 0.13 \\
\hline Sensation seeking & $-->$ & EIU & 0.20 & 0.01 & 0.13 \\
\hline Skills & $-->$ & EIU & 0.06 & 0.01 & 0.10 \\
\hline Time Online & $-->$ & EIU & 0.27 & 0.01 & 0.19 \\
\hline Engagement & $-->$ & EIU & 0.06 & 0.00 & 0.12 \\
\hline
\end{tabular}

Note: All coefficients significant at $\mathrm{p}<.01$.

Coefficients that were not significant were fixed to zero. 
Table 3 Standardized explanatory power of different variables in path models on excessive Internet use

\begin{tabular}{lc|crr} 
& $\mathrm{R}^{2}$ & Total & Direct & Indirect \\
\hline Age & & 0.26 & 0.07 & 0.19 \\
Gender & & 0.04 & 0.02 & 0.02 \\
Education & 0.02 & & 0.02 \\
Self-efficacy & 0.07 & 0.00 & -0.04 & 0.04 \\
Emotional problems & 0.01 & 0.20 & 0.19 & 0.01 \\
Sensation seeking & 0.06 & 0.17 & 0.13 & 0.04 \\
Time online & 0.16 & 0.19 & 0.19 & \\
Skills & 0.35 & 0.10 & 0.10 & \\
Breadth of engagement & 0.28 & 0.12 & 0.12 & \\
\hline Excessive Internet Use & 0.23 & & & \\
\hline
\end{tabular}

Note: All coefficients significant at $\mathrm{p}<.01$. 
Excessive Internet Use: Psychological Vulnerability and Digital Literacy 35

Figure 1 Model combining socio-demographic and psychological explanations of EIU

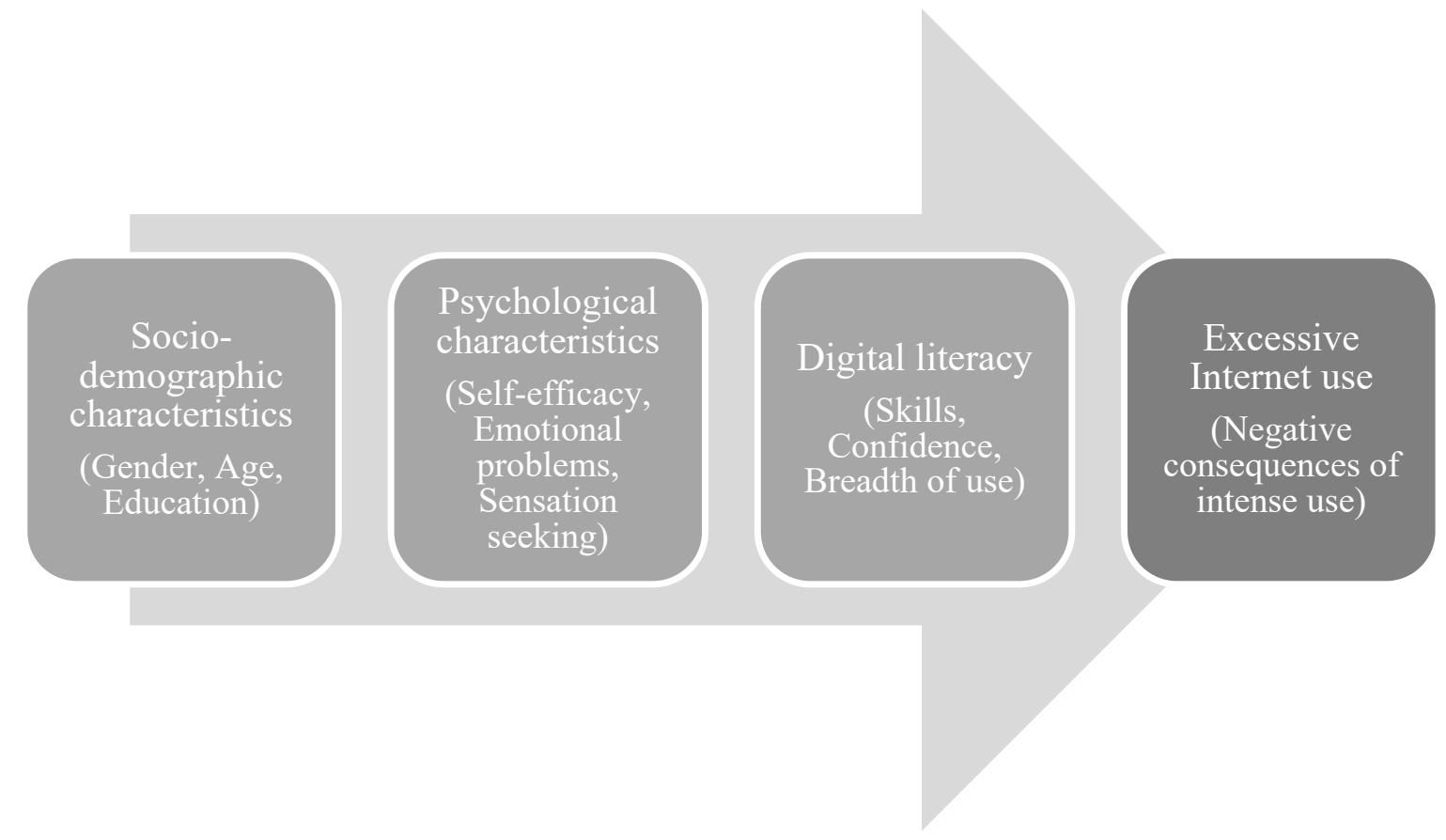


Figure 2 Predicted values of excessive Internet use for different levels of significant interaction effects between skills, emotional problems (EMP) and self-efficacy (SE).

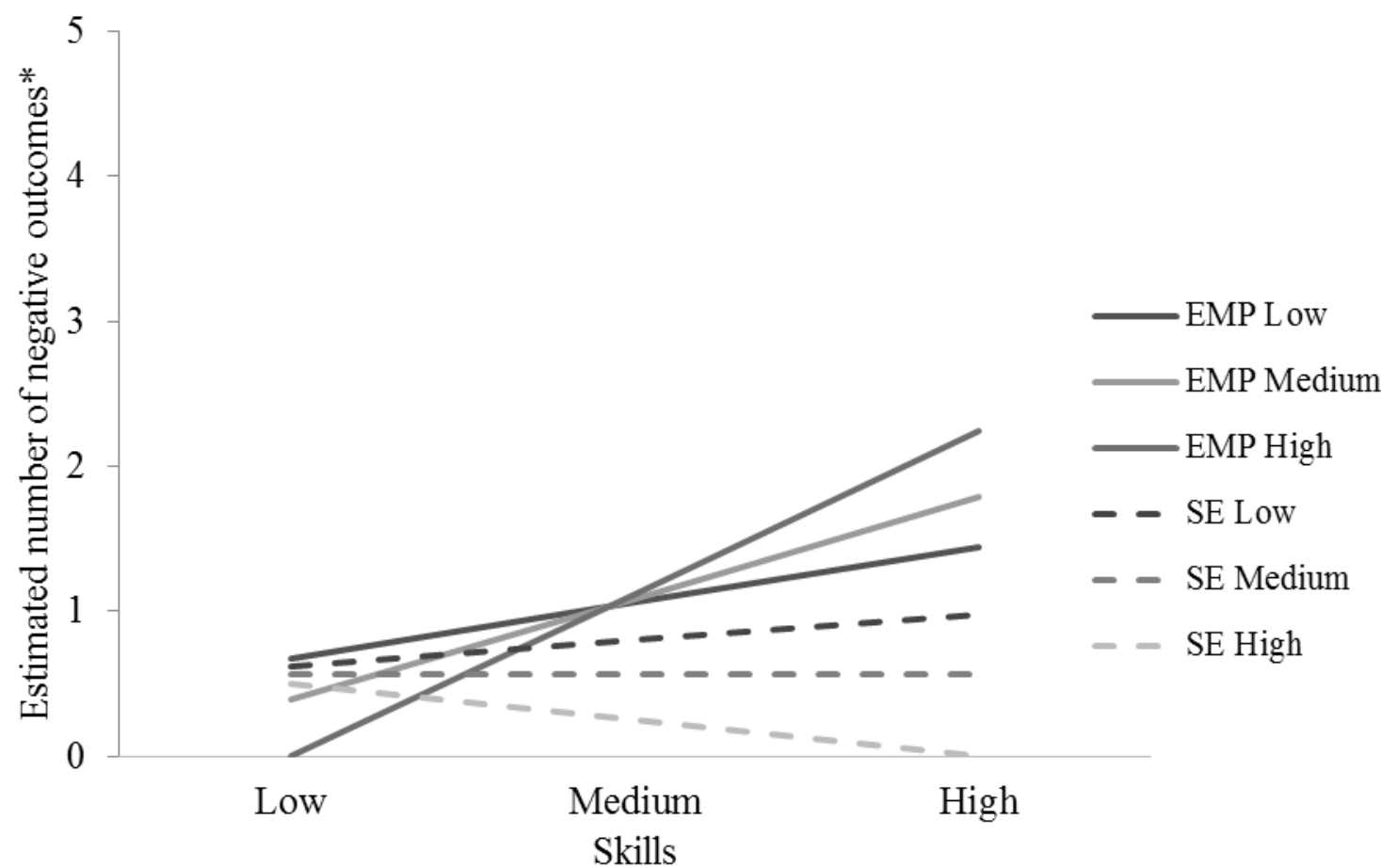

* Graph based on predicted negative outcomes and lowest value fixed to zero. All other variables held constant $(0)$.

Note: Values for emotional problems (EMP) and self-efficacy (SE) were 1, 2, and 3. Values for skill were 1, 4, and 8. 
${ }^{i}$ Path modeling assesses the relative strength of direct and indirect relationships of variables with the dependent variables. It can determine whether a model such as the one shown in Figure 1 can explain the pattern of correlations in the data, since it allows the researcher to fix certain relationships to zero and others to vary. The direction of the paths proposed in the model is determined by theoretical assumptions; significance of the coefficients and model fit do not indicate causality. The statistical program SPSS AMOS21 was used to test the hypothesized paths from skills to engagement. 